\title{
Priority based power sharing scheme for power consumption control in smart homes
}

\author{
Saher Umer, Yasuo Tan, Azman Osman Lim
}

Japan Advanced Institute of Science and Technology (JAIST), Ishikawa, Japan

\begin{abstract}
Efficient power consumption, intelligent power monitoring and interactive control of power consumption in smart homes are the key factors of sustainable development, reduction of power demand peaks and activities related to avoidance of blackout effects. The highlight of the proposed system is its ability to control maximum consuming power of the home appliances (HAs) and keep the total power consumption below a certain limit and allowing the home user more flexibility to operate their HAs based on HA priority. The proposed smart power management system consists of smart electric sensors (SESs) and power provisioning controller (PPC). The PPC and SES are concurrently used to control and maintain stability and complete power waveform behavior examination. However, we need to reassign an amount of consuming power from one HA to another when the ON/OFF status of HA changes. This paper offers a system to control the maximum power consumption considering heterogeneous HAs with different response speed. In order to guarantee the maximum consuming power limit, we compute the limited suggested power level for HAs. Simulation results show the effectiveness of our system in managing maximum power consumption.
\end{abstract}

Keywords: Power provisioning controller, smart electric sensor, maximum consuming power, home appliance priority, power supply or demand

\section{Introduction}

Smart homes is a place that equipped with information technology and computing, and can accept as well as response resident's request. The purpose is to provide resident a comfort, convenient, safety, and joyful life through managing various technologies at home [1]. Smart homes is a thought that supports control of several different systems in a household (e.g., heating, air conditioning, security, lighting, and audio/video systems) is acknowledged under the term smart homes [2]. In this way, the worst condition of energy shortage could happen due to increasing number of consumer electronics and their attractive functions according to the advancement of smart homes [3]. These increased power consumption resources have been forcing the human to search methods for managing power to reduce power consumption in home because power used in the home is a typical example of consumptive one. The high power required by home appliances (HAs) makes our homes one of the most critical areas for the impact of power consumption. As the residential areas are one of the main power consumers today, reducing power in home would contribute significantly to the environment. As more and more HAs and consumer electronics are connected, the power consumption in home area (i) tends to increase and (ii) leads an increase in the risk of power blackout [4]. As a result, an intelligent power management system is needed in smart homes, which is responsible for observing and handling the working operation of HAs [5].

Son and Moon [6] state that a home energy management system (HEMS) is the technology to manage and balance the home energy use. The HEMS plays a vibrant role in recognizing the efficient and versatile control of consuming power among HAs in smart homes. Pipattanasomporn, et al. [7] quote that the HEMS is a networked system and it is responsible for monitoring and managing the working

\footnotetext{
* Manuscript received September 3, 2013; revised November 4, 2013.

Corresponding author. Tel.: 81-90-2839-786; E-mail address: saher@ jaist.ac.jp.
} 
operation of HAs, and helping smart homes to reduce power consumption based on the specific set of requirements. However, nowadays the HEMS is a display-type of device that contains high processing unit to monitor and control the energy management in the home environment. On the other hand, the introduction of Smart Meter (SM) technologies is incorporated into the home gateway, which is totally different from other research works, e.g., in [8]. Therefore, in this research we assume that a smart electric sensor (SES) is proposed and used. The real time monitoring and measuring of power consumption levels of HAs through SES is a key and an integral part of smart homes. In our proposed scenario of the smart power management system the HAs considered to have different priority levels.

The higher priority HAs can take more power as compare to the lower priority HAs from the available power. However the change in ON/OFF status of the HAs, we may need to reassign a part of consuming power from lower priority HAs to the higher priority HAs. In this paper, we study the power assignment scheme for the HAs so that the total power is shared among HAs. To balance the power consumed and supply of the HAs, two types of controlling schemes are required. The first is the priority rule for the HAs that controls the power flows to HAs taking into account the limitation of the HA priority. The second is the controlling of total power consumption of the HAs below the power limit. This paper focus on reducing the power consumption until the specified limit reached, that operates on a single power source. We consider grid power supply, because this is the most popular power in a current household.

The rest of this paper is organized as follows. Research background and motivation that are related to this paper summarized in Section II. In Section III and IV, we described the system model and our proposed priority based power sharing scheme, respectively. In Section V, we define the overshoot control by using the limited suggested power level. In Section VI, we describe the simulation results and discussions. Finally, we conclude our research and future works in Section VII.

\section{Research Background and Motivation}

Electricity as an easy to use power is one of the most generally used power all over the world. Electric power system is the basic requirement for daily life and its failure will possibly paralyze the smart homes. The severe accidents by a large scale blackout have been well documented and many struggles have been done for avoiding such a large scale blackout [9], [10], [11]. On contrary, the blackout in smart homes is also a serious problem, but very less investigation study has been done for blackout prevention for home. It causes mental stress for the home user, and makes the smart homes unstable. The future smart homes should be stable completely. For this purpose, we need to introduce smart power management system for smart homes that shows a dynamic role in attaining reduction in extreme consuming power.

In order to reduce power consumption, it is essential to know how power is consumed. Consequently, power consumption monitoring is needed. The real time checking of instantaneous consuming power of the HAs can be positively achieved by SES [12]. Secondly, it is compulsory to manage and control the HAs to apply power reduction schemes. For controlling and managing the HAs, a number of HEMS were suggested and established [13]-[14]. The previous HEMS monitors and controls the home devices, and show home energy consumption information. HEMS in [15], is clarified with its complete goals that must be satisfied by HEMS and also offer strategies for the system growth to reduce power consumption at home. In general, priority of the HAs is also an important study in HEMS [16], but transient behaviour problems are ignored in various papers. Nevertheless, the existing research works about HEMS have been realized that it is infeasible to be deployed for smart homes. Therefore, we introduce a novel system, called smart power management system, which comprises of two important entities; a power provisioning controller (PPC) and a smart electric sensor. The PPC is used to collect ON/OFF status from each HA and instantaneous consuming power level from SES, and to send control signal back to each HA. Communication delay and communication interval between SES and PPC are also important factors in system stability and performance criteria that we should consider as well. The existing study shows the communication between HAs, SES and PPC but the consideration of communication interval and communication delay is uniqueness of this paper. The existing research studies consider the appliance priority but complete analysis of power waveform is main contribution of this paper. 


\section{System Model}

Main breaker of the house is the breaker that the feeder wire connects to. Main breaker is designed to trip if the amperage rating exceeds the value labeled on the breaker. Main breaker is further divided into sub-breakers. Sub-breakers are used to bound feed for branch circuit in house whole area. From there, the sub-breaker connects to outlets, lighting and other loads.

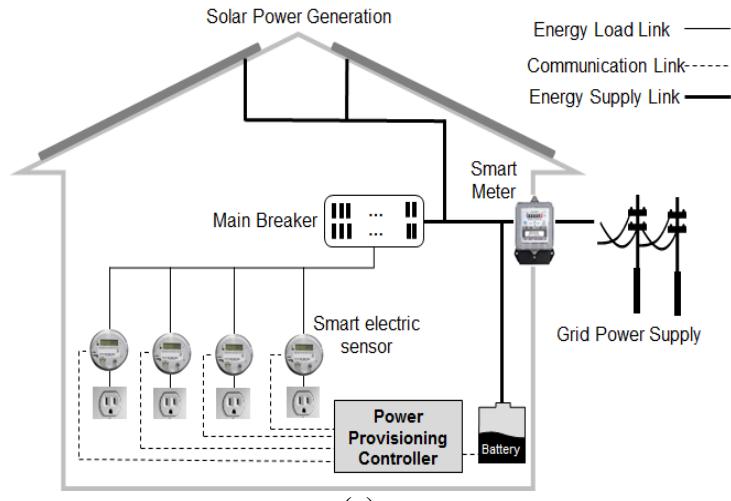

(a)

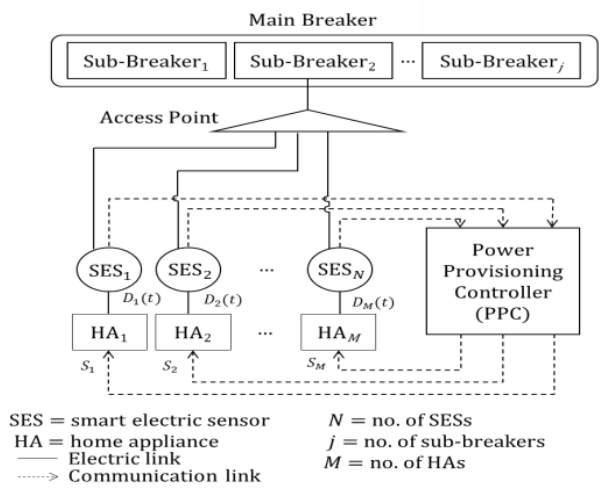

(b)

Fig. 1. (a) A sustainable energy house model with source and loads (b) System model.

In this paper, we assume that the HA is a smart device, which changes its power consumption level upon receiving the "suggested power message (SPM)" from the PPC, that performs intelligent computing upon receiving the information from the SESs. In this paper, we also assume that the SES is connected in between the sub-breaker and any single outlet, or single home equipment. SESs are responsible for sending “instantaneous consuming power message (ICPM)" to PPC.

The proposed system is to implements a control algorithm for maximum power consumption of HAs based on HA priority so that total power consumption does not exceed a certain power limit. The proposed system model consists of HAs, $m=\left\{H A_{1}, \ldots, H A_{M}\right\} \in M, n=\left\{S E S_{1}, \ldots, S E S_{N}\right\} \in N$ and a PPC, where $M=N$. An example of such a system is illustrated in Fig. 1(b). In our proposed system model, the total available power for $M$ HAs is denoted as $P$, thus remaining power $R$ can be represented as:

$$
R(t)=P-\sum_{i=1}^{M} S_{i}(t)
$$

$S_{i}(t)$ is the suggested power level sent to the HA by PPC in SPM message. The instantaneous consuming power level of $i t h \mathrm{HA}$ at time $t$ is given by $D_{i}(t)$ which is modeled by the exponential equation:

$$
D_{i}(t)=\left(D_{i}(t-\tau)-S_{i}(t)\right) \cdot e^{-a_{i} \cdot(\tau)}(t)+S_{i}(t)
$$

The parameter $a_{i}$ decides the response speed of that appliance. We additionally consider delay $(\tau)$ which is the time from the SES sends an ICPM message to PPC till the HA receives the SPM from PPC. It consists of SES transmitting time for $\left(t_{I C M P}\right)$ and its propagation delay ICPM $\left(\tau_{I C M P}\right)$, processing time at $\operatorname{PPC}\left(t_{P R O C}\right)$, PPC transmitting time of SPM $\left(t_{S P M}\right)$ and its propagation delay $\left(\tau_{S P M}\right)$.

$$
\tau=t_{I C M P}+\tau_{I C M P}+t_{P R O C}+t_{S P M}+\tau_{S P M}
$$

The function of PPC is to collect ON/OFF status from each HA and instantaneous consuming power from SES. After gathering information, using with HA priority, PPC computes the suggested power level for each HA. Upon receiving a SPM message from PPC, each HA behaves according to suggested power level. Our system works with message exchanges between HAs, SES and PPC. For this purpose, we 
define an interval MI (Monitoring Interval) of sequential communications between HAs, SESs and PPC.

In Fig. 2(b), an interval starting at $t$ and ending at $t+1$ is called the monitoring interval $(M I)$. The interval starts by the PPC sending a synchronization message $\left(t_{S Y N C}\right)$ to SESs then the message exchange between HAs, PPC and SES as mentioned above. We also assume that the maximum consuming power level $D_{i}^{\max }$ for each HA, given priority of each HA and system parameter $a_{i}$ would be specified with positive values in our proposed system. In general, the proposed system is a novel smart power management system that intelligently manages power flows among HAs based on their priorities. If a home user wants to use a HA (i.e., switches on the HA), the HA will send a "power request message" to PPC. Then PPC suggests the power level for the HA. If PPC assigns power equal to the maximum consuming power level of the HA then HA is said to be "satisfied" completely.

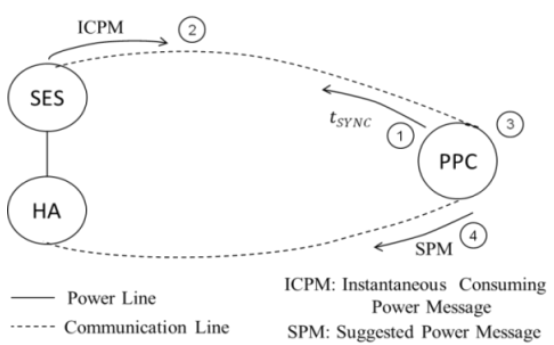

(a)

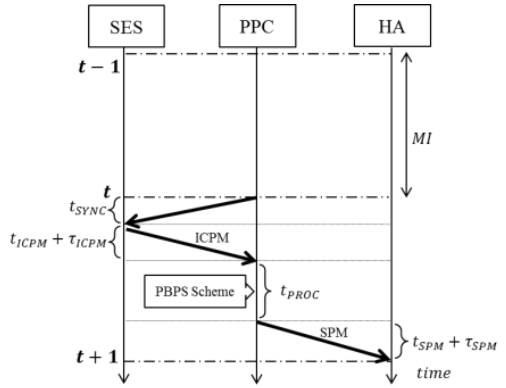

(b)

Fig. 2. Message procedures (a) operation (b) exchange.

\section{Priority Based Power Sharing Scheme}

The center notion of our proposed system is priority of a HA. HAs priority is used to share total available power among all active (attached) HAs. This is because some of HAs is more important than the others in home. To satisfy this significant criterion, a priority is given to each HA. At first, the system will choose the higher priority HAs and suggests higher satisfaction power level for them as compared to lower priority HAs. To calculate suggested power level for each HA, we propose a priority based power sharing (PBPS) scheme, which allows the home user to operate their HAs according to HA priority. Initially, a home user assigns given priority $G_{p r i}$ for each HA. In our proposed scheme, we use two other types of priorities; calculated priority $\left(C_{p r i_{i}}\right)$ and normalized priority $\left(N_{p r i_{i}}\right)$. The first $\left(C_{p r i_{i}}\right)$ changes with each iteration according to the ON/OFF status and HA satisfaction power level based on its maximum consuming power level, $\left(D_{i}^{\max }\right)$, it can be represented as:

$$
C_{\text {pri }_{i}}(t)= \begin{cases}0, & \text { if } S_{i}(t)=D_{i}^{\max } \\ G_{\text {pri }_{i},} & \text { if } S_{i}(t)<D_{i}^{\max }\end{cases}
$$

The second priority is called, normalized priority $\left(N_{p r i}\right)$ which can be calculated as:

$$
N_{\text {pri }_{i}}(t)=\frac{C_{p r i}(t)}{\sum_{i=1}^{M} C_{p r i}(t)}
$$

If $\sum_{i=1}^{M} C_{p r i}(t)=0$, states either all HAs are OFF or have been completely satisfied with their maximum consuming power level. If $\sum_{i=1}^{M} C_{p r i}(t)>0$, then some HAs are still not completely satisfied and PPC will keep tuning the suggested power level, $S_{i}(t)$ as:

$$
S_{i}(t)=\left\{\begin{array}{cl}
D_{i}^{\max }, & \text { if } R(t) \cdot N_{\text {pri }}(t)+S_{i}(t) \geq D_{i}^{\max } \\
R(t) \cdot N_{\text {pri }_{i}}(t)+S_{i}(t), & \text { if } R(t) \cdot N_{\text {pri }_{i}}(t)+S_{i}(t)<D_{i}^{\max }
\end{array}\right.
$$


The initial value of the suggested power level $S_{i}(0)$ is assumed zero. The initial value of remaining power $R(0)$ can be deduced from Eq. (1) which would be equal to the total available power $P$. In each iteration, the tuned suggested power level is refined and PPC re-compute the calculated priority, the normalized priority and remaining power. If $R(t)=0$, states that there is no more power to distribute among HAs and some HAs cannot be totally shared. In this situation HA will receive this final suggested power level. If $R(t)>0$ and $\sum_{i=1}^{M} C_{p r i}(t)>0$, the PPC need to keep tuning $S_{i}(t)$ until $R(t)=0$ or $\sum_{i=1}^{M} C_{\text {pri }_{i}}(t)=0$, and send last $S_{i}(t)$ to the HAs.

\section{PBPS Scheme with Overshoot Control}

In order to solve the overshoot problem observed with PBPS scheme, we propose a maximum consuming power control that is designed to guarantee the total power consumption below the specified limit. The $D_{i}(t)$ will be modified as:

$$
D_{i}(t)=\left(D_{i}(t-\tau)-L s_{i}(t)\right) \cdot e^{-a_{i} \cdot(\tau)}(t)+L s_{i}(t)
$$

In this algorithm, the suggested power level defined in the previous section $\left(S_{i}(t)\right)$ is considered as initial suggested power level, while the limited suggested power level for each interval $\left(L s_{i}(t)\right)$ can be calculated as in Fig. 3. Where, $E R(t)$ (estimated remaining power) is calculated as:

$$
E R(t)=\sum_{i=1}^{M} \max \left\{D_{i}(t-\tau), L s_{i}(t-1)\right\}
$$

PPC has to guess remaining power level based on the $D_{i}(t-\tau)$ and $L s_{i}(t-1)$ by taking their maximum. For sake of the robustness of our proposed system and ability to maintain the maximum power level, we use the estimated remaining power. The proposed modifications to control overshoot depend on three inputs: the suggested power level $\left(S_{i}(t)\right)$ from the previously proposed PBPS scheme, the limited suggested power level from previous interval $L s_{i}(t-1)$ together with the $D_{i}(t-\tau)$ from SES.

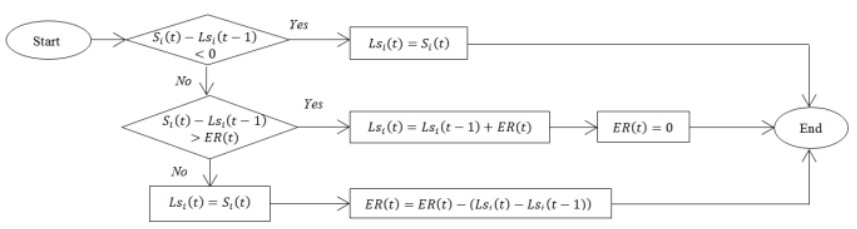

Fig.3. Calculation of limited suggested power $L s_{i}$.

Considering the case, new suggested power level is more than or equal to the old limited suggested power level. This situation reveals that the HA should increase power in the next interval, depending on the estimated remaining power level available. The PPC will set limited suggested power level as suggested power level. If the amount of increase is more than the estimated power level, PPC gives all the estimated remaining power to this HA and there would be no more power for the remaining HAs.

The case, when the new suggested power is less than the old limited suggested power level, means that the HA should decrease power in the next interval. In this case, PPC sets the limited suggested power level as suggested power level. The released power will not be used in this interval to provide the safety margin to avoid overshoot.

\section{Simulation Results and Discussions}

The objective of the simulation is to verify the system behavior and try to show that the power sharing criteria based on HA priority. For simulation scenario of our proposed system, we consider three HAs attached with three SESs. Throughout our simulation, both the delay time $(\tau)$ and synchronization time $\left(t_{S Y N C}\right)$ are assumed to be 0.02 seconds. The maximum consuming power levels for all three HAs are same as $D_{i}^{\max }=7 \mathrm{~A}$ and total power available for all HAs is $P=15 \mathrm{~A}$. It is obvious that, the total power 
available for all HAs is not enough for three HAs to reach their maximum consuming power level at the same time. In Fig. 4(a), we can notice that all three HAs have same value of $a_{i}, D_{i}^{\max }$ and given priority $G_{p r i}$. We observe that all three HAs shared the available power equally. However, in Fig. 4(b), we consider the situation when $G_{p r i}$ is different for each HA. In this scenario, we observe that the HA with high priority takes more power from the available power (i.e., PPC satisfied the HA more).

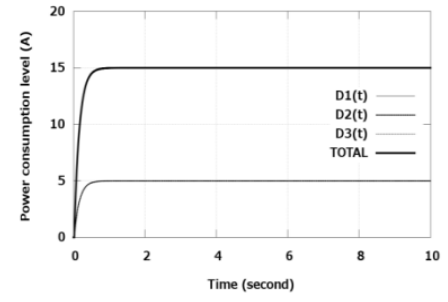

(a)

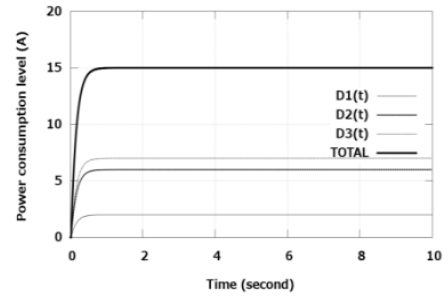

(b)

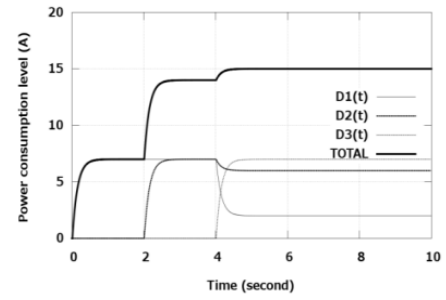

(c)

Fig. 4. Power consumption behavior of $H A s$ with $a_{i}=7$ (a) same priority for $H A s G_{p r i}=5$ (same turn ON time) (b) different priority for $\mathrm{HAs}$ are $\mathrm{G}_{\mathrm{pri}_{1}}=1, \mathrm{G}_{\mathrm{pri}_{2}}=3$ and $\mathrm{G}_{\mathrm{pri}_{3}}=7$ respectively (same turn ON time) (c) different priority for HAs are $\mathrm{G}_{\text {pri }_{1}}=1, \mathrm{G}_{\text {pri }_{2}}=3$ and $\mathrm{G}_{\mathrm{pri}_{3}}=7$, respectively, with turn ON time of all three HAs are: $\mathrm{HA}_{1}$ is $\mathrm{t}=0, \mathrm{HA}_{2}$ is $\mathrm{t}=2$ and $\mathrm{HA}_{3}$ is $\mathrm{t}=4$.

Next, we investigate the effect of the turning ON time of the HAs to the total available power sharing as depicted in Fig. 4(c), 5(a) and 5(b). In Fig. 4(c), before turning ON the higher priority HA, the already running lower priority HAs are assigned and reached high power level; the remaining power will be unsatisfactory for the newly turned ON high priority HA. In this case, PPC suggests the lower priority HAs to release some power for the high priority HA to reach better satisfaction level. We can notice that if the response speed for each HA is same, we can keep the maximum power limit. However, if the response speeds are different, the proposed PBPS scheme cannot guarantee maximum power limitation. At time $t=4 \mathrm{~s}$ overshoot is visible. Since the rate of change of instantaneous consuming power level of a HA which is turned ON later is faster than the rate of change of instantaneous consuming power level of a HA which is losing power for the higher priority HA, we have overshoot (as shown in Fig. 5(a)).

On the contrary, if the rate of change of instantaneous consuming power level of a HA which is turned ON later is slower than the rate of change of instantaneous consuming power level that is losing power, no overshoot is experienced (as shown in Fig. 5(b)).

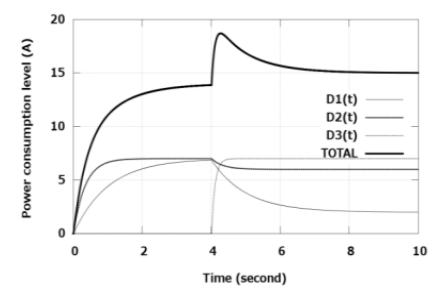

(a)

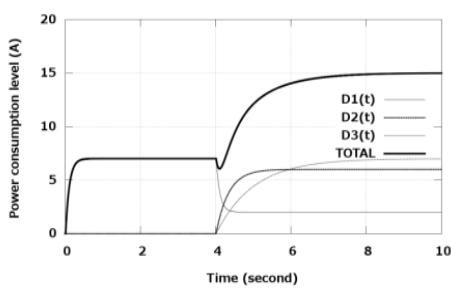

(b)

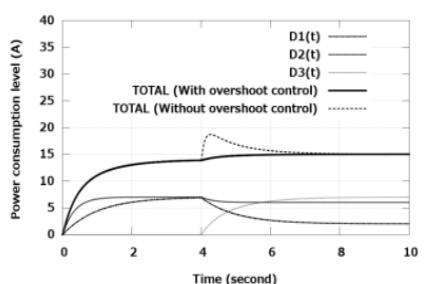

(c)

Fig. 5. Power consumption behavior of HAs (a) with $a_{1}=1, a_{2}=3, a_{3}=10$ with different priorities for HAs are $G_{p r i_{1}}=1, G_{\text {pri }_{2}}=3, G_{p r i_{3}}=7$, respectively, and turn ON time of all HAs are, $H A_{1}$ and $H A_{2}$ is $t=0$ and $H A_{3}$ is $t=4$ (b) with $a_{1}=10, a_{2}=3, a_{3}=1$ with different priorities for HAs are $G_{p r i_{1}}=1, G_{p r i i_{2}}=3, G_{p r i_{3}}=7$, respectively, and turn ON time of all HAs are, $H A_{1}$ is $t=0$ and $H A_{2}$ and $H A_{3}$ is $t=4$ (c) with $a_{1}=1, a_{2}=3$, $a_{3}=10$ with different priorities for $\mathrm{HAs}$ are $\mathrm{G}_{\mathrm{pri}_{1}}=1, \mathrm{G}_{\mathrm{pri}_{2}}=3, \mathrm{G}_{\mathrm{pri}_{3}}=7$, respectively and turn ON time of $\mathrm{HA}_{1}$, $\mathrm{HA}_{2}$ is $\mathrm{t}=0$ and $\mathrm{HA}_{3}$ is $\mathrm{t}=4$.

As we can see from the simulations, we cannot guarantee maximum power limitation with PBPS scheme. So, we introduce the concept of limiting the reassignment of remaining power by proposing 
PBPS scheme with overshoot control. In Fig. 5(c), comparison is shown between the system with and without overshooting control (PBPS scheme) using the same parameters as in Fig. 4(c).

It is clear that the proposed overshooting control kept the maximum consuming power level below the total available power limit by delaying the saturation of the newly turning ON HA.

\section{Concluding Remarks}

In summary, the smart homes can be defined as a residence equipped with computing and information technology which anticipates and responds to the needs of the occupants, working to promote their comfort, convenience, security and entertainment through the management of technology within the home. The efficiency, reliability and stability of the smart homes can be significantly improved via our proposed PPC and SESs are the important parts of smart power management system.

The use of SES measures the instantaneous consuming power level from the HAs. The functions of PPC are to gather ON/OFF status from each HA, instantaneous consuming power $D_{i}(t)$ from SESs and calculate the remaining power for HAs. The remaining power level is calculated based on estimation technique which is the key factor to control critical behavior of our proposed system. With this estimation, we can guarantee the maximum power consumption below the total available power limit. We also analyzed our proposed system in simulation environment. The simulation results reveal that the dependency of system parameters $a_{i}$ (HA response speed), $D_{i}^{\max }$ (maximum consuming power level) and $G_{p r i}$ (HA given priority by user). We noticed the critical system behavior such as overshoot in total power consumption.

Our future work is to investigate the priority based power sharing scheme for the proposed system in a real home environment via a computer simulation. Besides that, we also look into different power supplies, such as the solar panel, the electric vehicle, and the fuel cell.

\section{References}

[1] Frances KH. Smart Homes: Past, Present and Future, In: Inside the Smart Home, Springer-Verlag, London, 2003.

[2] Marusic L, Skocir P, Petric A, Jezic G. Home-in-palm a mobile service for remote control of household energy consumption. in Proc. of Int. Conf. on Telecommun. (ConTEL), 2011:109-116.

[3] Hong L, Jyh CC, Ming CJ, Ching YH. Integration of GPRS and wireless LANs with multimedia applications. Adv. in Multimedia inform. Process.-Lecture Notes in Comp. Sci., 2002; 2532:704-711.

[4] Mario JK, Christian R, Wolfgang K. An intelligent knowledge representation of smart home energy parameters. Energy EndUse Efficiency Issues (EEE) World Renewable Energy Congress, 2011:921-928.

[5] Jinsoo H, Chang SC, Wan KP, Ilwoo L. Green home energy management system through comparison of energy usage between the same kinds of home appliances. IEEE Int. Symp. on Consumer Electron. (ISCE), 2011:1-4.

[6] Young SS, Topi P, Kyeong DM. Home energy management system based on power line communication. IEEE Trans. on Consumer Electron. (ICCE), 2010; 56(3):1380-1385.

[7] Manisa P, Murat K, Saifur R. An algorithm for intelligent home energy management and demand response analysis. Trans. on Smart Grid, 2012; 3(4):2166-2173.

[8] Arman K, Anuradha A. The effect of a smart meter on congestion and stability in a power market. IEEE Conf. on Decision and Control, 2010:194-198.

[9] J. Barkans, and D. Zalostiba, New concept and solutions for prevention of power system blackout. Int. Conf. on Development in Power System Protection (DPSP), April 2010: 1-5.

[10] Jekabs B, Diana Z. Blackout prevention and power system self-restoration. IEEE Int. Conf. on Comp. as Tool, 2007:1547 1554.

[11] Zhong S, Sun Z. Challenges and opportunities in emergency management of electric power system blackout. Int. Conf. on EProduct E-Service and E-Entertainment (ICEEE), 2010:1-4.

[12] ITU-T Focus group Smart Grid WGI, Use Cases Deliverable, 2010.

[13] Jinsoo H, Chang SC, Ilwoo L. More efficient home energy management system based on Zigbee communication and infrared remote controls. IEEE Trans. on Consumer Electron., 2011; 57(1):85-89.

[14] Chia HL, Ying WB, Hsien CC, Chi HH. Home appliance energy monitoring and controlling based on power line communication. Int. Conf. on Consumer Electron. (ICCE), 2009:1-2.

[15] Rossello B, and Ana J. Towards efficient energy management: defining HEMS and smart grid objectives. Int. J. on Advances in Telecommun., 2011;4(3\&4):249-236.

[16] Takekazu K, Kenji Y, Takashi M. Energy on demand: efficient and versatile energy control system for home energy management. IEEE Int. Conf. on Smart Grid Commun., 2011:392-397. 